\title{
Broadening of mm-wave beam by plasma turbulence in the basic plasma physics device TORPEX and TCV tokamak
}

\author{
Ivo Furno $^{(1)}$, Stefano Alberti ${ }^{(1)}$, Marcelo Baquero ${ }^{(1)}$, Oulfa Chellai ${ }^{(1)}$, Tim P. Goodman $^{(1)}$ Paolo Ricci $^{(1)}$, \\ Fabio Riva ${ }^{(1)}$, Omar Maj ${ }^{(2)}$ \\ (1) Swiss Plasma Center, Ecole Polytechnique Fédérale de Lausanne, Switzerland; e-mail: ivo.furno@epfl.ch \\ (2) Max Planck Institute for Plasma Physics, Garching, Germany
}

Concerns have recently been raised about loss of efficiency in Neoclassical Tearing Modes (NTM) stabilization by Electron Cyclotron (EC) due to broadening of mm-wave beams interacting with plasma turbulence at the edge and the scrape-off layer (SOL) of tokamak plasmas. This has stimulated a number of experimental and theoretical studies on well diagnosed basic plasma physics devices and tokamaks. The SOL, characterized by open field lines, is populated by field-aligned structures, dubbed "blobs", driven by the underlying turbulence. An externally injected mm-wave beam interacting with blobs is scattered, leading to a broader time-averaged beam profile at the targeted absorption location. On next-generation fusion devices, such as ITER, this would lead to a reduced efficiency of NTM stabilization that may require modulation of the beam to recover the required performance.

This paper reports on direct measurements of mm-wave power scattering through a turbulent plasma in the basic plasma device TORPEX and the TCV tokamak. In both devices, a dedicated experimental setup allows measurements of the transmitted mm-wave beam for comparison with first-principle numerical simulations of plasma turbulence and mm-wave propagation.

In TORPEX [1], a simple magnetized toroidal device, we review first direct experimental measurements of the scattering of mm-wave beams by previously diagnosed plasma blobs. Here, the wavelength of the beam is comparable to the characteristic size of the blob. We use Langmuir probe measurements to show that fluctuations of the electron density induce correlated fluctuations in the transmitted power. These fluctuation amplitudes are in agreement with predictions from a first-principles full-wave model, which uses conditionally sampled 2D electron density profiles to model the dielectric tensor in a cold plasma approximation [2].

In TCV, we show first experimental evidence, in a tokamak plasma, that fluctuations of the transmitted mm-wave power are caused by SOL turbulence. The distance from the TCV launcher to the detector is $\sim 1.7 \mathrm{~m}$, similar to that from the SOL to the $\mathrm{q}=2$ surface in ITER. The measured fluctuations are consistent with predictions of mm-wave beam transmission when mm-wave scattering by SOL turbulence is included in the wave propagation model. These predictions are based on first-principle simulations of the SOL turbulence using the GBS code [3]. This provides correlation lengths and the level of density fluctuations that are used in a parametric statistical model of the turbulence by the WKBeam code [4]. We can thus calculate the expected beam transmission through the plasma to a synthetic detector as in the experiment, confirming experimentally the broadening strength. Furthermore, the present results are a benchmark for the WKBeam code as a tool to correctly simulate beam broadening, and thus the associated current drive delocalisation, in future tokamaks, provided verified input parameters such as those from GBS can be made available.

[1] I. Furno et al., J. Plasma Phys. 81, 345810301 (2015).

[2] O. Chellai el., Phys. Rev. Lett. 120, 105001 (2018).

[3] A. Snicker et al, Nucl. Fusion 58, 016002 (2018).

[4] P. Ricci et al., Plasma Phys. Controlled Fusion 54, 124047 (2012). 